\title{
Novel polyrotaxane cross-linkers as a versatile platform for slide-ring silicone
}

Tran, Jakob-Anhtu; Madsen, Jeppe; Skov, Anne Ladegaard

\section{Published in:}

Bioinspiration \& Biomimetics

Link to article, DOI:

10.1088/1748-3190/abdd9f

Publication date:

2021

Document Version

Peer reviewed version

Link back to DTU Orbit

Citation (APA):

Tran, J-A., Madsen, J., \& Skov, A. L. (2021). Novel polyrotaxane cross-linkers as a versatile platform for slidering silicone. Bioinspiration \& Biomimetics, 16, [025002]. https://doi.org/10.1088/1748-3190/abdd9f

\section{General rights}

Copyright and moral rights for the publications made accessible in the public portal are retained by the authors and/or other copyright owners and it is a condition of accessing publications that users recognise and abide by the legal requirements associated with these rights.

- Users may download and print one copy of any publication from the public portal for the purpose of private study or research.

- You may not further distribute the material or use it for any profit-making activity or commercial gain

- You may freely distribute the URL identifying the publication in the public portal

If you believe that this document breaches copyright please contact us providing details, and we will remove access to the work immediately and investigate your claim. 
ACCEPTED MANUSCRIPT

\section{Novel polyrotaxane cross-linkers as a versatile platform for slide-ring silicone}

To cite this article before publication: Jakob-Anhtu Tran et al 2021 Bioinspir. Biomim. in press https://doi.org/10.1088/1748-3190/abdd9f

\section{Manuscript version: Accepted Manuscript}

Accepted Manuscript is "the version of the article accepted for publication including all changes made as a result of the peer review process, and which may also include the addition to the article by IOP Publishing of a header, an article ID, a cover sheet and/or an 'Accepted Manuscript' watermark, but excluding any other editing, typesetting or other changes made by IOP Publishing and/or its licensors"

This Accepted Manuscript is @ 2020 IOP Publishing Ltd.

During the embargo period (the 12 month period from the publication of the Version of Record of this article), the Accepted Manuscript is fully protected by copyright and cannot be reused or reposted elsewhere.

As the Version of Record of this article is going to be / has been published on a subscription basis, this Accepted Manuscript is available for reuse under a CC BY-NC-ND 3.0 licence after the 12 month embargo period.

After the embargo period, everyone is permitted to use copy and redistribute this article for non-commercial purposes only, provided that they adhere to all the terms of the licence https://creativecommons.org/licences/by-nc-nd/3.0

Although reasonable endeavours have been taken to obtain all necessary permissions from third parties to include their copyrighted content within this article, their full citation and copyright line may not be present in this Accepted Manuscript version. Before using any content from this article, please refer to the Version of Record on IOPscience once published for full citation and copyright details, as permissions will likely be required. All third party content is fully copyright protected, unless specifically stated otherwise in the figure caption in the Version of Record.

View the article online for updates and enhancements. 


\title{
Novel polyrotaxane cross-linkers as a versatile platform for slide-ring silicone
}

\author{
Jakob-Anhtu Tran ${ }^{1}$, Jeppe Madsen ${ }^{1}$ and Anne Ladegaard Skov ${ }^{1}$ \\ ${ }^{1}$ Danish Polymer Center, Department of Chemical and Biochemical Engineering, Technical University \\ of Denmark, Søltofts Plads, Building 227, 2800 Kgs. Lyngby, Denmark \\ E-mail: jakant@kt.dtu.dk
}

Received xxxxxx

Accepted for publication $\mathrm{xxxxxx}$

Published xxxxxx

\begin{abstract}
Slide-ring elastomers have garnered a lot of interest for their potential use in dielectric elastomer actuators due to their intrinsically soft nature and high elasticity. However, the use of sliding cross-linkers has been constrained by their low miscibility with commonly used elastomer precursors and the specialized curing chemistries that are necessary for incorporating them into networks. Here, we have presented a method to produce vinyl functional polyrotaxane cross-linkers that are compatible with polysiloxanes and can be processed by industrially scalable methods. The sliding silicone films that were fabricated with these novel cross-linkers were highly extensible $(>350 \%)$ and did not exhibit strain hardening even at high elongation. The composite films also retained the favorable dielectric properties of silicone elastomers such as the characteristic low dielectric loss. The modified polyrotaxanes present a robust platform for producing a new class of sliding silicone elastomers with well-defined networks structures.
\end{abstract}

Keywords: Silicone, slide-ring elastomer, polyrotaxane, dielectric elastomer actuator, DEA

\section{1. Introduction}

Dielectric elastomer actuators (DEAs) present a lot of promise in advancing the field of soft robotics and biomimetic 4 design due to their soft and flexible nature, high deformability and lightness in weight [1-3]. Given these inherent characteristics, DEAs offer the potential to emulate the energy 7 transducing characteristics of natural muscles and opening the 8 door to novel technologies that cannot be realized with traditional motors such as self-cleaning surface coatings and tunable elastomeric lenses [4,5]. While commercially available elastomers have been considered as suitable candidate materials for DEAs, there has been a lot of progress in tailoring elastomers specifically for actuation, namely softer materials and/or materials with higher dielectric permittivity. The approaches range from creating composite systems to completely novel chemical compositions [6-8] and network structures $[9,10]$.
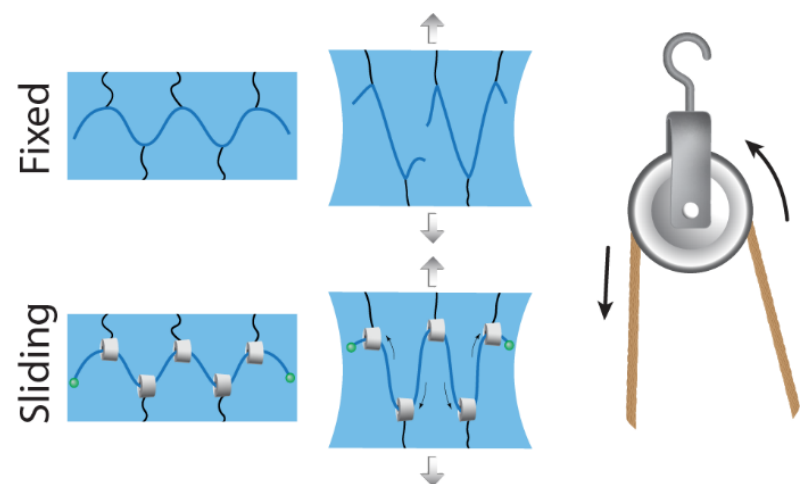

Figure 1: The pulley-like behavior of sliding networks

In contrast to conventional elastomers, slide-ring materials demonstrate a novel type of dynamic network structure consisting of mobile cross-links that can rearrange on a molecular scale. The sliding links are formed by ring molecules that have been threaded on linear axis polymers. 
1 This type of supramolecular assembly allows the rings to act as cross-links that can slide on the constrained axis (as seen in Figure 1), thus imparting the networks with an additional degree of entropic freedom [11]. These sliding elastomers have been proven peculiar in their intrinsic softness while maintaining high deformability and toughness [12]. This reversible and soft nature of slide-ring materials makes them ideal candidates for dielectric elastomer actuators provided that they can be designed to be electrically robust too.

Polyethylene glycol (PEG) and $\alpha$-cyclodextrin $(\alpha-C D)$ based polyrotaxanes have shown promise as a versatile platform for slide-ring materials due to their commercially available components and relative ease of production. While the production of PEG/ $\alpha-C D$ polyrotaxanes has reached a commercial scale, their inclusion in silicone networks has been limited $[13,14]$. The slide-ring materials that have been utilized for DEA fabrication have so far been restricted to poly- $\varepsilon$-caprolactone grafted systems, which have a relatively high elastic modulus ( $1 \mathrm{MPa})$ compared to silicone elastomers[15-17]. The use of polysiloxanes or other network chemistries with polyrotaxane cross-linkers is generally restricted by the low reactivity of the pure polyrotaxanes as well as immiscibility issues [18]. Both issues are caused by the dense hydrogen bonding that occurs between the hydroxyl groups of the threaded rings and can be solved by chemical modification of the polyrotaxanes.

Here, we present a synthetic pathway to produce vinyl functional slide-ring cross-linkers that can be processed with common solvents and incorporated into polysiloxane networks through hydrosilylation. This type of curing chemistry is ubiquitous in silicone elastomers due to its high efficiency and selectivity as well as ease to work with [19]. These features are important in ensuring the versatility and scalability of the slide-ring cross-linkers as the basis of a new class of sliding silicone elastomers.

The slide-ring silicone elastomers that were produced with the novel cross-linkers exhibited high softness while maintaining the favorable dielectric properties that are characteristic for silicones. Additionally, the sliding elastomers possessed a highly time dependent relaxation mode resulting from the sliding of links within the polyrotaxane that is not present in traditional silicone networks. This phenomenon demonstrates the possibility of designing more intricate dielectric elastomer transducers with two distinctive modes of behavior determined by the operational speed of the system.

\section{2. Experimental}

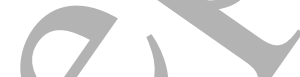

\subsection{Materials}

Polyethylene glycol (PEG 35000) was purchased from 50 Fluka. (benzotriazol-1-yloxy)tripyrrolidinophosphonium hexafluorophosphate (PyBOP) was purchased from Tokyo Chemical Industry Co., Ltd. 1,3-divinyl-1,1,3,3tetramethyldisilazane, platinum-divinyltetramethylsiloxane complex $(3.0 \% \mathrm{Pt})$ as well as hydride and vinyl terminated polydimethylsiloxanes (PDMS M were procured from Gelest Inc. Household bleach (Klorin Original, 5\% available chlorine) was purchased from a local supplier (Salling group A/S). Sylgard ${ }^{\circledR} 184$ elastomer kit was purchased from Dow Chemical Company Ltd. and Ecoflex ${ }^{\mathrm{TM}}$ 00-30 elastomer kit was purchased from FormX Amsterdam. All other chemicals were purchased from Merck KGaA and used as received unless otherwise stated.

\subsection{Characterization}

${ }^{1} \mathrm{H}-\mathrm{NMR}$ spectra were acquired on a Bruker $300 \mathrm{MHz}$ spectrometer in either deuterated dimethyl sulfoxide (DMSO) or chloroform at ambient temperature unless stated otherwise, with the residual solvent peaks used as the internal standard. Size Exclusion Chromatography (SEC) measurements were conducted with Shimadzu instrumentation (SIL-10AD autoinjector, LC-10AD liquid chromatograph, RID-10A refractive index detector) with a $10 \mathrm{mM} \mathrm{LiBr}$ DMSO eluent flow of $1.000 \mathrm{~mL} / \mathrm{min}$ at room temperature through a Millipore/Waters Ultrastyragel $10^{5} \AA$ column.

Electrical breakdown tests were performed on an in-house built device based on international standards (IEC 60,243-1 (1998) and IEC 60,243-2 (2001)) [20]. A stepwise increase of voltage was applied to the samples $\left(50 \mathrm{~V} \mathrm{step} \mathrm{s}^{-1}\right)$ at a rate of 0.5 steps $\mathrm{s}^{-1}$. Film thickness was determined through optical microscopy of the film cross-section.

Dielectric relaxation spectroscopy (DRS) was performed at room temperature and low electrical field $\left(\sim 1 \mathrm{~V} \mathrm{~mm}^{-1}\right)$ on a Novocontrol Alpha-A high-performance frequency analyser (Concept 40 Top Class System, Novocontrol Technologies $\mathrm{GmbH} \& \mathrm{Co}$, Germany) operating in the frequency range $10^{-1}-10^{6} \mathrm{~Hz}$. The samples were approximately $0.1 \mathrm{~mm}$ thick and $20 \mathrm{~mm}$ in diameter.

Differential scanning calorimetry (DSC Q1000; TA Instruments) measurements were performed on the elastomer samples from -90 to $200^{\circ} \mathrm{C}$ at a heating rate of $10^{\circ} \mathrm{C} \mathrm{min}-1$ under nitrogen atmosphere.

Linear viscoelastic analysis (LVE) was conducted on a TA Discovery HR-1 rheometer with $20 \mathrm{~mm}$ diameter parallelplate geometry. The measurements were carried out at a fixed strain amplitude of $1 \%$ with a frequency range of 0.01 to $100 \mathrm{~Hz}$.

The tensile properties of the elastomers were measured using the Instron 3340 materials testing system (INSTRON, US). Samples of $30 \mathrm{~mm}$ length and $3.18 \mathrm{~mm}$ width were placed between two clamps with an initial separation distance of $20 \mathrm{~mm}$. Strain was defined as $\left(L-L_{0}\right) / L_{0}$ where $L$ is the distance between the clamps during stretching and $L_{0}$ the intial 
1 separation distance. The test specimens were elongated uniaxially at a strain rate range of 0.5 to $200 \mathrm{~mm} \mathrm{~min}^{-1}$.

Actuation measurements were performed by connecting the thin film samples (thickness of 110 to $150 \mu \mathrm{m}$ ) with a high voltage power supply (PS375, Stanford Research Systems Inc.) and applying step-wise increased voltages (500 V per step) until electrical breakdown. The samples were mounted with $20 \%$ pre-strain on a round plastic frame with an inner diameter of $30 \mathrm{~mm}$. Circular electrodes with a diameter of 10 $\mathrm{mm}$ were painted on either sides of the samples with carbon grease. The actuation was determined by video analysis software (Tracker).

\subsection{Preparation of polyrotaxanes}

To prepare the carboxylic acid terminated PEG-COOH, $10 \mathrm{~g}(0.29 \mathrm{mmol})$ of PEG was oxidized in $100 \mathrm{~mL}$ of DI water with 2,2,6,6-tetramethyl-1-piperidinyloxy radical (TEMPO, $100 \mathrm{mg}, 0.64 \mathrm{mmol}), \mathrm{KBr}(116 \mathrm{mg}, 0.97 \mathrm{mmol})$ and an aqueous $\mathrm{NaClO}$ solution ( $20 \mathrm{~mL}, 5 \%$ available chlorine) at a $\mathrm{pH}$ of 11-12 for $15 \mathrm{~min}$. The oxidation was quenched with $10 \mathrm{~mL}$ of ethanol. The reaction mixture was then acidified with $\mathrm{HCl}$ to a $\mathrm{pH}$ below 2. Proper ventilation was ensured by performing the procedure in a fume-hood with a lowered sash to avoid exposure to highly toxic chlorine gas. The product was extracted with two $100 \mathrm{~mL}$ aliquots of dichloromethane (DCM) that were then combined and evaporated in vacuo. The product was dissolved in $200 \mathrm{~mL}$ of hot $\left(60^{\circ} \mathrm{C}\right)$ ethanol and precipitated at $-20{ }^{\circ} \mathrm{C}$. The precipitate was then washed with ethanol and dried in vacuo overnight. The end-group conversion was confirmed with ${ }^{1} \mathrm{H}-\mathrm{NMR}$ to be $>98 \%$.

The polyrotaxanes were prepared as follows: $3 \mathrm{~g}$ (0.09 mmol, $0.18 \mathrm{mmol}$ carboxylic acid) of PEG-COOH and $13.7 \mathrm{~g}(12.3 \mathrm{mmol})$ of $\alpha$-cyclodextrin $(\alpha-\mathrm{CD})$ were dissolved in $100 \mathrm{~mL}$ of DI water and stirred for 15 minutes at room temperature before overnight complexation at $5{ }^{\circ} \mathrm{C}$. The complexed pseudo-polyrotaxane (PPR) was then freeze-dried and crushed into a fine powder. The PPR was then end-capped with the addition of 1-adamantylamine (169 mg, $1.1 \mathrm{mmol})$, PyBOP (581 mg, $1.1 \mathrm{mmol}$ ) and N, N-diisopropylethylamine $(0.2 \mathrm{~mL}, 1.2 \mathrm{mmol})$. The mixture was dissolved in $100 \mathrm{~mL}$ of dry N, N-dimethylformamide (DMF) and stirred overnight at $\mathrm{RT}$ in an inert atmosphere. The reaction mixture was then washed through centrifugation, twice with a 1 to 1 mixture of $\mathrm{DMF} / \mathrm{methanol}$ and twice with methanol. The obtained solid was then dissolved in dimethylsulfoxide and precipitated in water, followed by repeated washings with water and freezedrying.

\subsection{Preparation of vinyl functional polyrotaxanes (ViPR)}

48 The polyrotaxane $(1 \mathrm{~g}, 7.35 \mu \mathrm{mol})$ was dissolved in dry DMSO $(40 \mathrm{~mL})$ in a nitrogen atmosphere. A premade mixture ( $4.4 \mathrm{~g}, 20.4 \mathrm{mmol})$ of 1,3 -divinyl-1, 1,3,3- tetramethyldisilazane (DVTMDS) and 1,1,1,3,3,3hexamethylsilazane (HMDS) was slowly added to the reaction vessel in a drop-wise manner. The molar ratio of the silylating agents was varied between experiments. The reaction mixture was stirred for 6 hours at $60{ }^{\circ} \mathrm{C}$, followed by addition of dry toluene $(40 \mathrm{~mL})$. The mixture was then left to react for an additional 20 hours. The product was then precipitated in methanol $(800 \mathrm{~mL})$, washed with methanol and dried under vacuum, resulting in a white solid (1.2 $\mathrm{g}, 69 \%$ yield).

\subsection{Preparation of slide-ring silicone films}

Hydride terminated PDMS $\left(0.41 \mathrm{~g}, M_{n}=24000 \mathrm{~g} \mathrm{~mol}^{-1}\right)$, vinyl terminated PDMS extenders $(0.17 \mathrm{~g}$, $\left.M_{n}=20000 \mathrm{~g} \mathrm{~mol}^{-1}\right)$ and ViPR cross-linkers (0.06 $\mathrm{g}$, $f_{\text {vinyl }}=0.42 \mathrm{mmol} \mathrm{g}^{-1}$ ) were dissolved in toluene. Platinum catalyst was added (1.1 $\mathrm{mg}, 50 \mathrm{ppm})$ and the mixture was left to pre-cure for 10 minutes at $80^{\circ} \mathrm{C}$. The mixture was then cast into a Teflon mold and left to evaporate for a day at room temperature before the final overnight curing at $80{ }^{\circ} \mathrm{C}$. Pure PDMS control films were prepared with identical hydride terminated PDMS (1.4 g, $\left.M_{n}=24000 \mathrm{~g} \mathrm{~mol}^{-1}\right)$ and vinyl terminated PDMS extenders $\left(0.57 \mathrm{~g}, M_{n}=20000 \mathrm{~g} \mathrm{~mol}^{-1}\right)$ and vinyl functional polysiloxane cross-linkers $(0.14 \mathrm{~g}$, $\left.f_{\text {vinyl }}=0.61 \mathrm{mmol}^{-1}\right)$. The formulation was mixed on a FlackTek Inc. DAC 150.1 FVZ-K SpeedMixer ${ }^{\mathrm{TM}}$ and then coated on a glass substrate using a film applicator (3540 bird, Elcometer, Germany) with a $200 \mu \mathrm{m}$ blade. Commercial Sylgard®184 and EcoFlex ${ }^{\mathrm{TM}}$ 00-30 silicone films were prepared according to the instructions provided by the suppliers.

\section{Results and discussion}

\subsection{Vinyl functional slide-ring cross-linkers}

Polyrotaxanes consisting of polyethylene glycol (PEG) and $\alpha$-cyclodextrin ( $\alpha$-CD) were synthesized according to methods previously described in the literature [21], with minor modifications. An alternative coupling reagent was adopted instead of the commonly used benzotriazol-1yloxytris(dimethylamino)phosphonium hexafluorophosphate to avoid the formation of the carcinogenic byproduct hexamethylphosphoramide. Additionally, the end-capping reaction was performed at room temperature to inhibit esterification side-reactions that can form polyrotaxane dimers.[21] Size exclusion chromatography was employed to confirm that the threaded $\alpha$-CDs were trapped by the bulky capping groups at the end of the PEG chains, thus forming a stable supramolecular compound that does not disassociate when dissolved in DMSO. This can be seen in the decreased retention volume of the polyrotaxanes caused by the larger hydrodynamic size compared to the PEG and $\alpha-\mathrm{CD}$ precursors (Figure 2). The achieved polyrotaxane yield varied between 


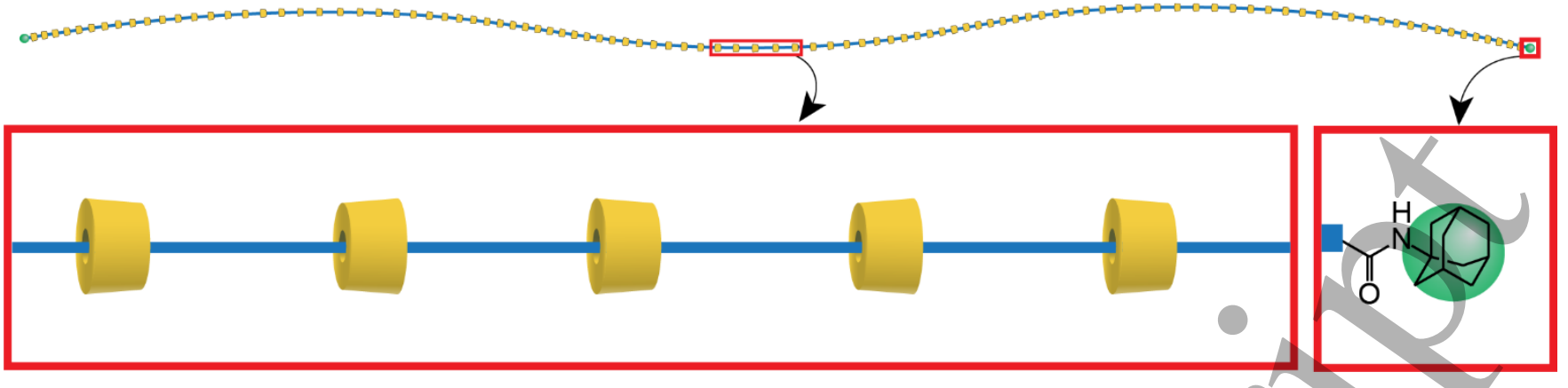

Figure 3: Approximate size of a polyrotaxane compound with $100 \alpha-C D$ rings that have been threaded on a PEG chain with a molecular weight of $35000 \mathrm{~g} \mathrm{~mol}^{-1}$. This relates to an inclusion ratio of $25 \%$ compared to the theoretical maximum of 400 rings.

160 to $70 \%$ based on PEG ( $\alpha-\mathrm{CD}$ was used in excess). The 2 average number of threaded rings per each PEG chain and the molecular weight of the polyrotaxanes were then determined by ${ }^{1} \mathrm{H}-\mathrm{NMR}$. The values were attained by integrating the spectral peaks assigned to the adamantane end-groups (labeled $a, b$ and $c$ in Figure 2) of the capped PEG chains and the H1 peak $(4.80 \mathrm{ppm})$ of the cyclodextrin glucose units (see Figure 2).

The degree of ring threading has an inverse relation to the length of the PEG chain [22]. It is thus possible to control the amount of threaded rings based on the molecular weight of the selected chain polymer. In this case, approximately 100 rings were threaded for each polyrotaxane molecule, which corresponds to a threading ratio of $25 \%$ compared to the maximum amount of rings that could be fit on the axis chains (as seen in Figure 3). The theoretical maximum is established based on the assumption that one ring fits two ethylene glycol units [23].

The spontaneous threading process is driven by the formation of hydrogen bonds between the cyclodextrin rings when they are aligned by the axis polymers [24]. The threaded complexes are further stabilized by arranging ordered 3 hexagonal packing structures with neighboring compounds
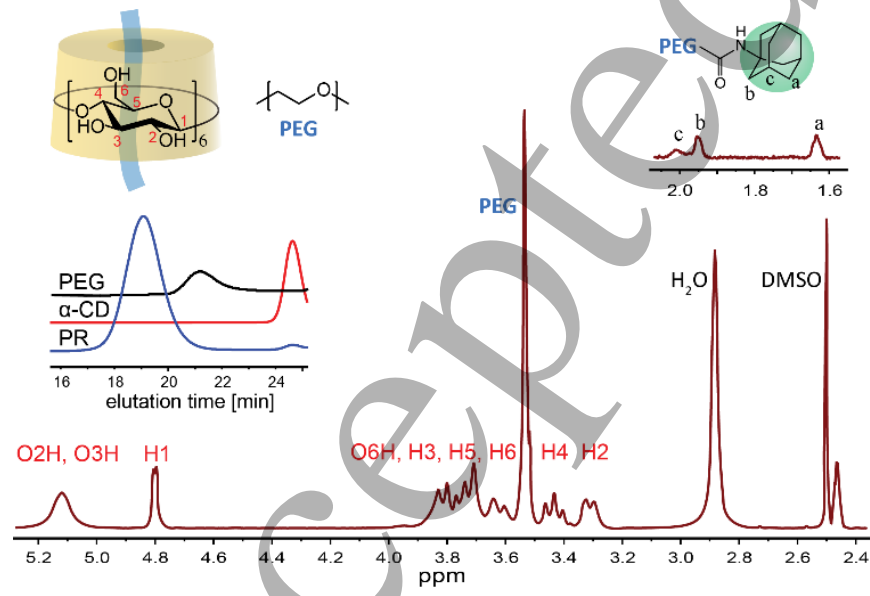

Figure 2: ${ }^{1} \mathrm{H}-\mathrm{NMR}$ spectrum $\left(300 \mathrm{MHz}, \mathrm{d}_{6}-\mathrm{DMSO}, 80{ }^{\circ} \mathrm{C}\right.$ ) and SEC analysis (detector: RI, eluent: $10 \mathrm{mM} \mathrm{LiBr}$ in DMSO) of polyrotaxanes. (illustrated in Figure 4) [25,26]. However, the abundant hydrogen bonding between the threaded cyclodextrin rings has been found to both limit the solubility of the final polyrotaxanes and lower the reactivity of threaded rings $[18,27]$. Additionally, the tendency for the rings to aggregate constricts the sliding phenomena by locking the rings in place [28]. Chemical modification of the threaded $\alpha-C D$ rings was thus necessary to improve the solubility of the compounds in organic solvents and to disrupt the dense intermolecular packing structure. It was also possible to introduce reactive functional groups that are necessary for network formation on the threaded rings during this process. The hydroxyl groups on the threaded $\alpha$-CD rings were substituted with different molar ratios of commercially available silylating agents HMDS and DVTMDS. The modified polyrotaxanes were prepared by slowly adding a premade mixture of the silylating agents into a rigorously stirred polyrotaxane solution in DMSO. After the initial reaction had progressed for 6 hours, toluene was added to the reaction mixture to increase the degree of modification by preventing the precipitation of the partly silylated polyrotaxanes. The conversion of the hydroxyl groups was confirmed by ${ }^{1} \mathrm{H}-\mathrm{NMR}$ (Table 1 ).

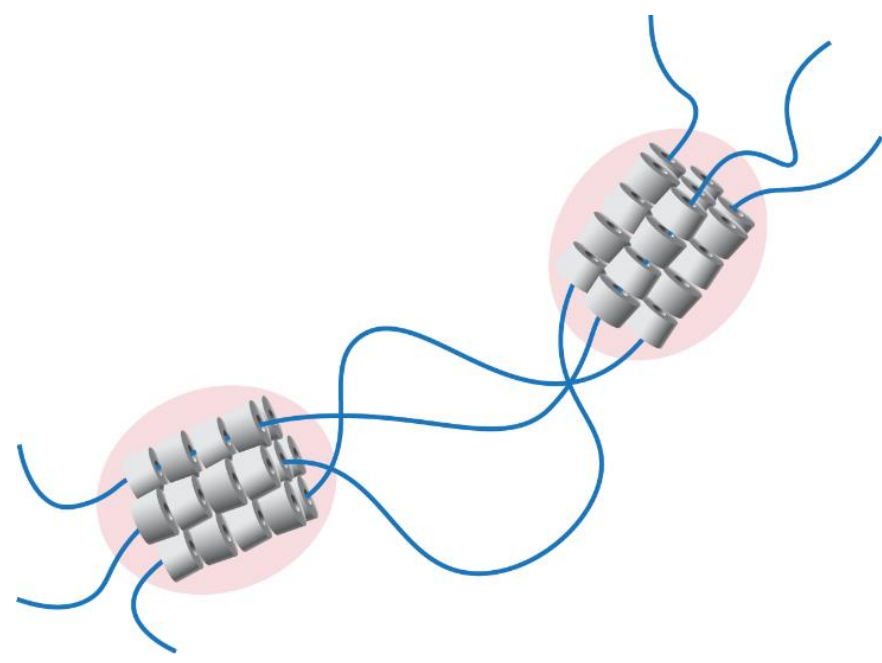

Figure 4: Packing structure of the threaded cyclodextrin rings

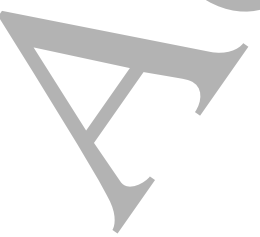



polyrotaxane (Table 1). The final polyrotaxane cross-linkers were soluble in industrially common solvents such as
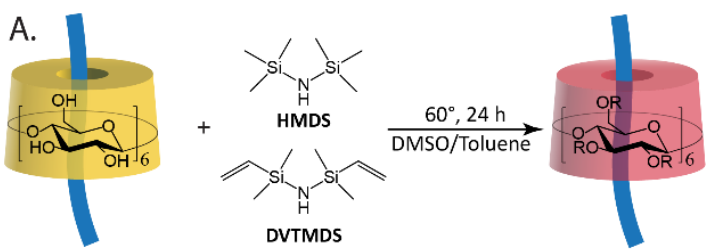

$\mathrm{R}=$

$\mathrm{H}$,
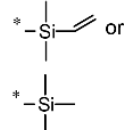

\section{C.}

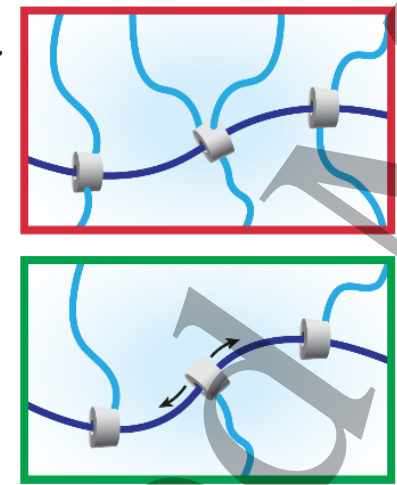

Figure 5: A) Synthesis of modified polyrotaxanes with vinyl functional rings (ViPR). B) Vinyl groups per $\alpha$-CD ring based on the feed ratio $C$ ) Fixed cross-links forming on the $\alpha-C D$ rings versus freely sliding network. chloroform, toluene, and tetrahydrofuran. The aforementioned solvents can be easily removed during processing thus making it possible to handle the modified polyrotaxanes by industrially scalable methods such as solvent casting. The introduction of vinyl groups allows the cross-linkers to be utilized in silicone networks by means of hydrosilylation curing chemistry, which is widely used in commercial silicone formulations due to its high efficiency and selectivity [19].

Table 1: Effect of the feed ratio of the vinyl functional DVTMDS silylating agent on the final functional group composition of the modified polyrotaxanes (ViPR), where TMS is the unreactive trimethylsilyl group and $\mathrm{OH}$ the residual hydroxyl groups. The degree of substitution is determined by the number of hydroxyl groups that were substituted per each glucose unit in the ring.

\begin{tabular}{c|ccc|c}
\hline \multirow{2}{*}{ Feed } & \multicolumn{2}{|c|}{ Groups per $\boldsymbol{\alpha}-\mathbf{C D}$ ring } & Degree of \\
& Vinyl & TMS & OH & substitution \\
\hline $\mathbf{0}$ & 0 & 12.9 & 5.1 & 2.15 \\
$\mathbf{0 . 2 5}$ & 0.6 & 12.0 & 5.3 & 2.11 \\
$\mathbf{0 . 3 5}$ & 1.0 & 10.5 & 6.6 & 1.91 \\
$\mathbf{0 . 5 1}$ & 2.1 & 11.2 & 4.7 & 2.21 \\
$\mathbf{0 . 7 3}$ & 5.3 & 8.1 & 4.6 & 2.24 \\
$\mathbf{1}$ & 11.0 & 0 & 7.0 & 1.83 \\
\end{tabular}

\subsection{Formulating slide-ring silicone elastomers}

The modified slide-ring cross-linkers were pre-cured in toluene with hydride terminated PDMS and chain extenders before solvent casting and final curing (see preparation scheme in Figure 6). The extent of the pre-curing reaction was found to be essential for ensuring a homogeneous reaction mixture in the final curing of the elastomer films. Insufficient pre-curing caused the formation of cross-linker rich domains (visible by the naked eye), while excessive pre-curing resulted in overabundant gelling in the casting solution that caused complications in obtaining even film geometries. Given the large molecular weight of the slide-ring cross-linkers $\left(\mathrm{M}_{\mathrm{n}}=230000 \mathrm{~g} \mathrm{~mol}^{-1}\right)$, longer linear PDMS segments were necessary to ensure that the polyrotaxane weight fraction would not be dominant in the curing formulations. Chain extenders were utilized to increase the length of the PDMS segments to approximately $64000 \mathrm{~g} \mathrm{~mol}^{-1}$, resulting in

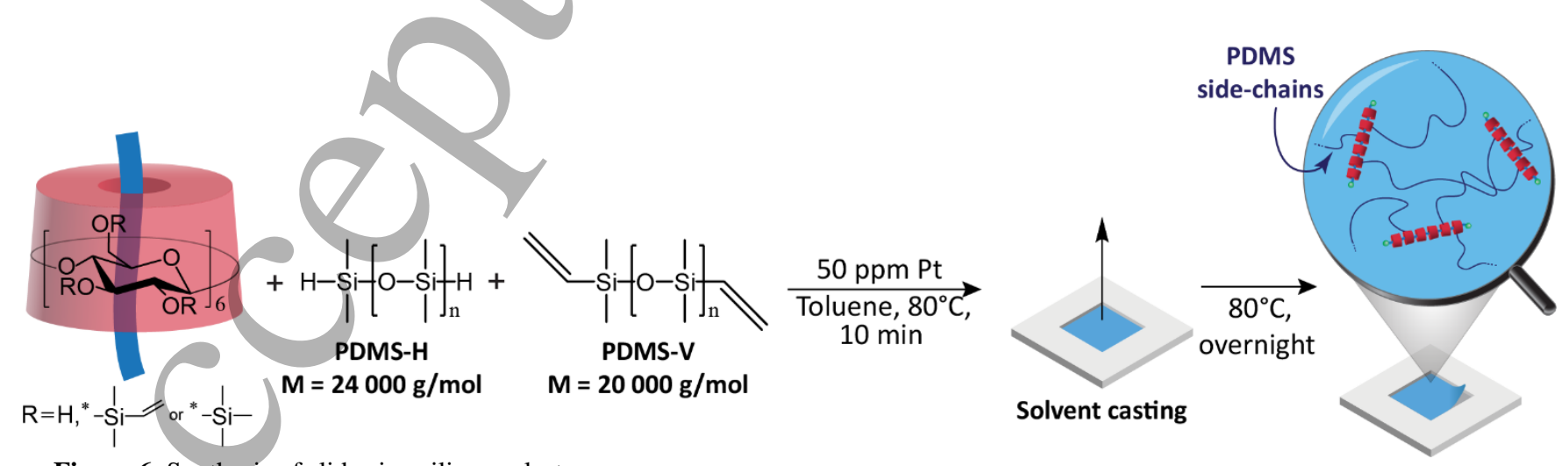

Figure 6: Synthesis of slide-ring silicone elastomers

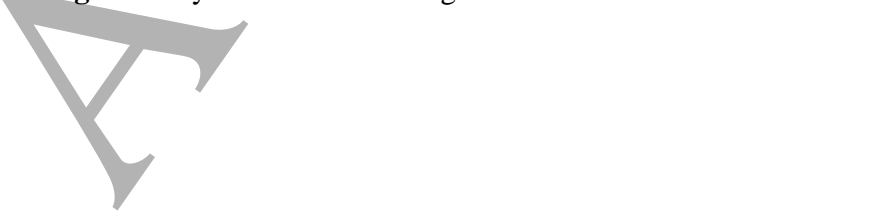


Table 2: Dielectric and mechanical properties of sliding films.

\begin{tabular}{|c|c|c|c|c|c|c|c|c|}
\hline & $\begin{array}{c}\text { Polyrotaxane } \\
\text { wt } \%\end{array}$ & $\mathbf{r}$ & $\mathbf{Y}_{5 \%} \mathbf{M P a}$ & $\begin{array}{c}\text { Tensile } \\
\text { strength } \\
\text { MPa }\end{array}$ & $\begin{array}{c}\varepsilon^{\prime} @ 0.1 \\
\mathbf{H z}\end{array}$ & $\begin{array}{l}\varepsilon^{\prime} @ 1 \\
\mathbf{M H z}\end{array}$ & $\begin{array}{c}\tan \delta \\
@ 0.1 \mathrm{~Hz}\end{array}$ & $\mathbf{E}_{b} \mathbf{V} / \mu \mathrm{m}$ \\
\hline & 9.4 & 1.5 & 0.529 & 0.75 & 2.7 & 2.8 & $8.8 \cdot 10^{-2}$ & 59.0 \\
\hline & 10.6 & 2.0 & 0.520 & 0.70 & 2.5 & 2.5 & $3.5 \cdot 10^{-2}$ & 65.5 \\
\hline & 14.8 & 2.5 & 2.017 & 0.77 & 2.6 & 2.7 & $1.2 \cdot 10^{-1}$ & 54.6 \\
\hline & 17.3 & 3.0 & 2.962 & 0.94 & 2.8 & 2.8 & $3.2 \cdot 10^{-2}$ & 56.3 \\
\hline & 19.4 & 3.5 & 5.251 & 1.16 & 3.0 & 2.9 & $1.7 \cdot 10^{-2}$ & 49.3 \\
\hline PDMS Control & 0 & 1.5 & 0.192 & 5.46 & 3.3 & 3.3 & $8.0 \cdot 10^{-2}$ & 39.5 \\
\hline Sylgard 184 & 0 & NA & 1.597 & 7.09 & 3.2 & 3.1 & $10=-2$ & 113.4 \\
\hline
\end{tabular}

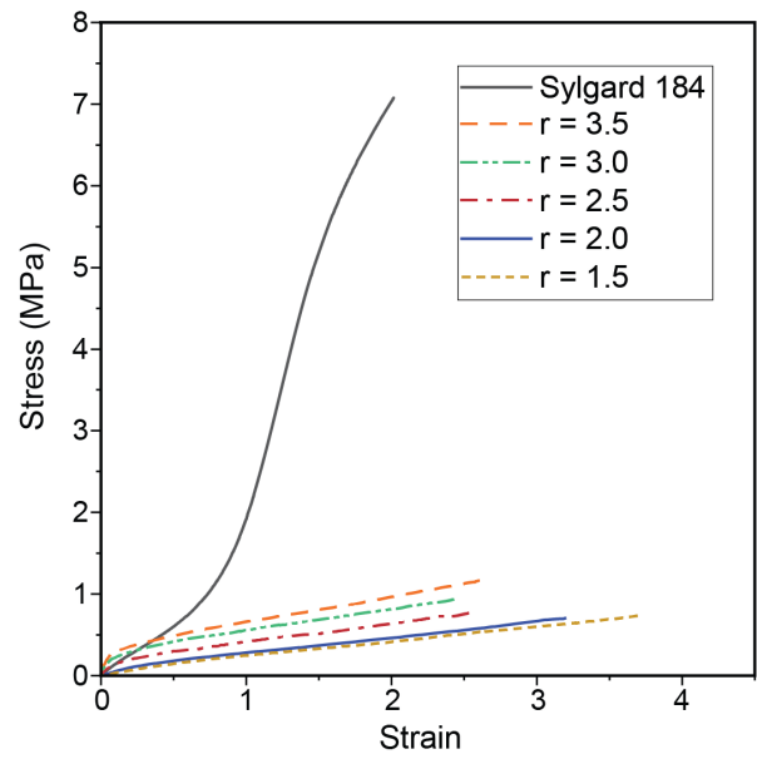

Figure 7: Tensile properties of sliding silicone films with differing cross-linker concentrations. Strain is defined as ( $\left.L-L_{0}\right) / L_{0}$ where $L$ is the distance between the clamps during stretching and $L_{0}$ the intial separation distance.

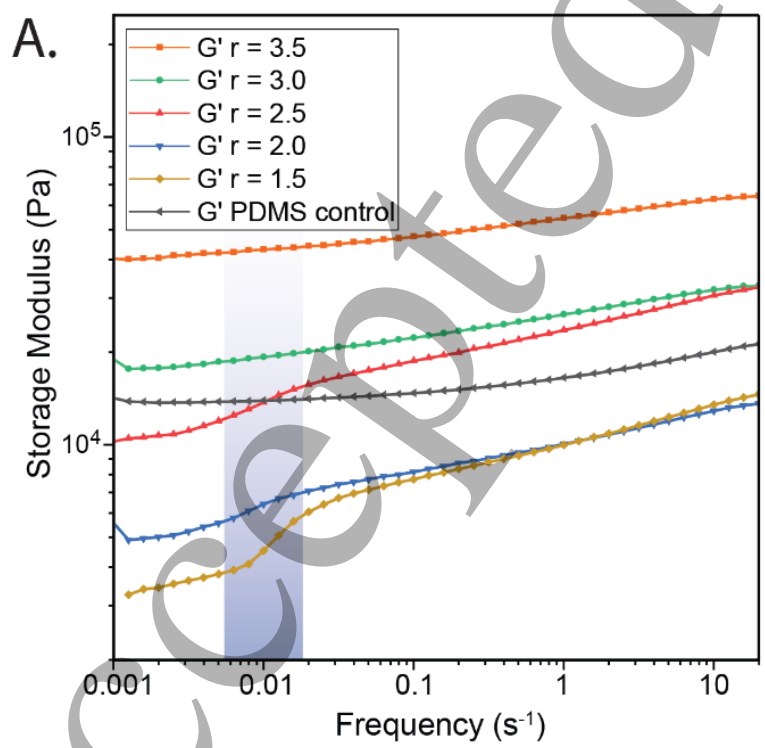

silicone compositions of 81 to 91 weight percent without compromising the stoichiometric balance between the crosslinking functional groups. The stoichiometric balance between the sliding cross-linkers and the silicone component was controlled by the ratio between the vinyl groups of the modified polyrotaxanes and the hydride end-groups of the linear PDMS (Equation 1).

$$
r=\frac{[\text { vinyl }]}{[\text { hydride }]}
$$

The cured elastomers were transparent, and no visible phase separation was observed under optical microscopy.

\subsection{Characterization of sliding silicone elastomers}

A series of sliding silicone samples was fabricated with various cross-linker concentrations. The elastic modulus, taken as the slope of the stress-strain curves at 5\% strain, $\left(\mathrm{Y}_{5 \%}\right)$ of the elastomers increased in line with the cross-linker loading while still exhibiting strains at break of over $200 \%$ (as seen in Figure 7). The sliding samples with a lower crosslinker fraction ( $\mathrm{r}=1.5$ and 2.0) had significantly lower elastic moduli compared to the commercial silicone elastomer reference sample. None of the sliding samples exhibited considerable strain hardening and maintained a stable stress-

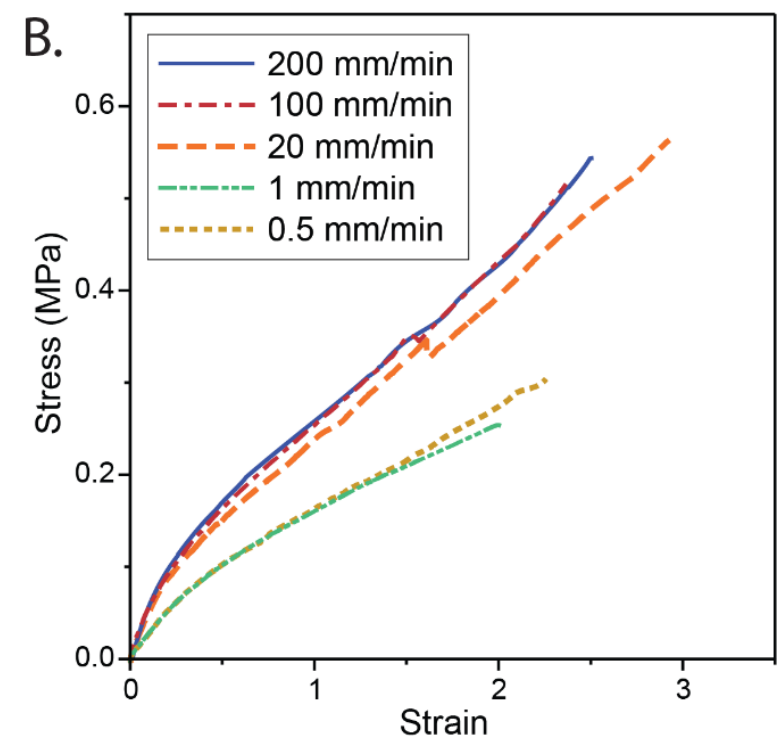

Figure 8: Mechanical properties of the slide-ring networks. A) LVE study of the sliding transition for different cross-linker concentrations. B) Tensile properties of $\mathrm{r}=1.5$ sample at different strain rates $\left(L_{0}=20 \mathrm{~mm}\right)$. All measurements conducted at room temperature. 
1 strain slope after the initial inflection point. The tensile strength of the samples did not vary significantly between the samples with a lower r-value of 1.5 to 2.5 , but increased incrementally at higher ratios (Table 2).

All glass transition temperatures of the sliding samples fell below the $-90{ }^{\circ} \mathrm{C}$ limit of the analytical setup used.

The dielectric properties of the sliding silicone elastomers were almost identical to the pure PDMS control sample (Table 2). No significant change in dielectric permittivity $\left(\varepsilon^{\prime}\right)$ or dielectric loss tangent ( $\tan \delta$ ) was observed with increasing cross-linker fractions. This can be contributed to the change in dielectric properties of the polyrotaxane cross-linkers after chemical modification. The substitution of the polar hydroxyl groups on the threaded cyclodextrin rings effectively lowers both the permittivity and dielectric loss of the polarotaxanes cross-linkers resulting to yield properties with great similarity to PDMS. The dielectric breakdown strength $\left(\mathrm{E}_{\mathrm{b}}\right)$ of the sliding samples was considerably lower than the commercial control sample, but this decreased value is most likely due to the absence of reinforcing silica fillers in the prepared formulations.

\subsection{Time dependent behavior and sliding transition}

The rheological properties of the sliding silicone elastomers were studied by linear viscoelastic analysis to get an understanding of the relaxation mechanisms within the networks. A distinct transition in the storage modulus was observed for the three sliding samples with a lower crosslinker concentration ( $\mathrm{r}=1.5$ to 2.5 ) around a frequency of $0.01 \mathrm{~Hz}$ (Figure 8A) (in other words a relaxation time of approximately $100 \mathrm{~s}$ ). This time dependent transition is credited to the onset of ring sliding. Thus, the sliding entropy of the rings dominates the elastic response of the elastomers at slower operational speeds. This phenomenon is not common in conventional elastomers, as seen for the PDMS control sample. The frequency range of the transition was not significantly affected by the cross-linker concentration, which indicates that the time-scale is not affected by the stiffness of the elastomers within the given range of samples. Rather we hypothesize that a certain degree of relaxation within the polyrotaxane needs to take place before free sliding is possible. This phenomenon has been previously observed in slide-ring hydrogels that exhibit a time-dependent transition between a rubbery and sliding state [31]. To our knowledge, a similar transition has not been reported for dry slide-ring elastomers to date.

No transition could be observed in the two samples with a higher cross-linker concentration ( samples $r=3.0$ and $r=3.5$ ). The absence of ring sliding could be due to the stiffening effect of the high cross-linker loading suppressing the slope of the transition. Higher polyrotaxane weight fractions in the curing formulations have been found to have a reinforcing effect on the cured elastomers that is independent from concentration of networking groups (Table S1). The reinforcing effect is also considered to be responsible for the significant increase in elastic modulus of the samples with a higher cross-linker concentration.

The time-dependent properties of the sliding elastomers were confirmed by extensional rheology. No significant change in elastic modulus or stress strain profile could be observed when extension rate was decreased from 200 to $20 \mathrm{~mm}$ per minute $\left(L_{0}=20 \mathrm{~mm}\right)$. However, the modulus drops significantly by half from 0.6 to $0.3 \mathrm{MPa}$, when the rate is lowered to $1 \mathrm{~mm}$ per minute or less (Figure 8B). This type of strain rate dependent phenomena is not present in conventional elastomers (Figure S4).

The average Hencky strain rate (defined as $\left.\dot{\varepsilon}_{H}=(d L / d t) / L\right)$ was determined to compare the time-scale of the extended samples with the sliding transition observed in the LVE study. The calculations confirm that the samples with the stiffer elastic response were extended faster $\left(\overline{\dot{\varepsilon}}_{H}=0.05\right.$ to $\left.0.40 \mathrm{sec}^{-1}\right)$ than the sliding threshold while the softer samples were extended at rate below the sliding transition $\left(\overline{\dot{\varepsilon}}_{H}=0.004\right.$ and $0.003 \mathrm{sec}^{-1}$ ).

The results affirm the existence of two distinct modes of behavior depending on if the threshold for sliding has been crossed. This presents the potential to design smart dielectric elastomer transducers with two distinct actuation responses determined by the operational speed of the system.

To evaluate the actuation potential of the novel sliding silicone films, the figure of merit $\left(F_{o m}\right)$ was calculated based on Equation 2 where $\varepsilon_{0}$ is the vacuum permittivity. The expression for the figure of merit was developed to present a single parameter to directly compare the actuation performance of different materials [32].

$$
F_{o m}=\frac{3 \varepsilon^{\prime} \varepsilon_{0} E_{b}^{2}}{Y}
$$

Table 3: Actuation comparison for sliding silicone elastomers (SSE).

\begin{tabular}{lccccc}
\hline & $\mathbf{Y}_{\mathbf{5} \%} \mathbf{M P a}$ & $\boldsymbol{\varepsilon}^{\prime} @ \mathbf{a . 1 H z}$ & $\mathbf{E}_{\mathbf{b}} \mathbf{V} / \boldsymbol{\mu m}$ & $\begin{array}{c}\text { Actuation strain by } \\
\text { area (\%) }\end{array}$ & Fom \\
\hline SSE & $0.308^{*}$ & 2.7 & 59.0 & 20.3 & 0.81 \\
Sylgard 184 & 1.597 & 3.2 & 113.4 & 17.5 & 0.68 \\
Ecoflex 00-30 & 0.306 & 3.4 & 42.7 & 21.8 & 0.52 \\
PDMS Control & 0.192 & 3.3 & 39.5 & 30.4 & 0.71
\end{tabular}

*Elastic module of SSE when the strain rate is lower than the inverse sliding transition time 
The sliding silicone elastomers were compared with commercial silicone elastomers, Sylgard 184 and Ecoflex 0030 , that are commonly used for fabricating acuating devices. Furthermore, simple actuation tests were conducted with all of the samples. Samples were pre-strained by $20 \%$ and actuation tests were conducted by appling a voltage on the samples at intervals of increasing strength (at $500 \mathrm{~V}$ steps) until breakdown. The actuation strain was measured by video analysis.

The figure of merit values and maximum actuation strain of the samples can be seen in Table 3 . The sliding actuators exhibited increased actuation at lower voltages and a moderate increase in $F_{\text {om }}$ when compared to the reference samples. The sliding elastomers performed considerably better in regards to breakdown strength when compared to the softer Ecoflex 0030. The PDMS control sample resulted in the best actuation performance but it was obvious that the elastomer suffered from hysteresis. These initial results indicate the potential of the sliding system as a versatile platform for preparation of soft DEAs with improved crosslinking compared to commercially available soft elastomes based on low crosslinking densities, such as Ecoflex 00-30.

\section{CONCLUSION}

In summary, vinyl functional polyrotaxane cross-linkers were produced with commercially available precursors. The novel cross-linkers are soluble in common solvents and can be cured by conventional techniques, thus increasing their processability by industrially scalable methods and allowing them to be incorporated in silicone elastomer systems. This opens up the possibility to explore a new class of slide-ring silicone elastomers. The sliding silicone films had a welldefined network structure and exhibited two distinct timedependent elastic responses that could be observed by both linear viscoelastic analysis and extensional rheology.

\section{Acknowledgements}

This work was supported by DTU's alliance stipend program.

\section{References}

[1] Gu G Y, Zhu J, Zhu L M and Zhu X 2017 Bioinspiration and Biomimetics 12011003

[2] Brochu P and Pei Q 2010 Macromol. Rapid Commun. 31 1036

[3] Skov A L and Yu L 2018 Adv. Eng. Mater. 201700762

[4] Bauer S, Bauer-Gogonea S, Graz I, Kaltenbrunner M, Keplinger C and Schwödiauer R 2014 Adv. Mater. 26 149-62

[5] Maffli L, Rosset S, Ghilardi M, Carpi F and Shea H 2015 Adv. Funct. Mater. 25

[6] Racles C, Cozan V, Bele A and Dascalu M 2016 Des. Monomers Polym. 19 496-507
[7] Madsen F B, Yu L, Mazurek P S and Skov A L 2016 Smart Mater. Struct. 25

[8] Opris D M 2018 Adv. Mater. 301703678

[9] Madsen F B, Daugaard A E, Hvilsted S and Skov A L Macromol. Rapid Commun. 37 378-413

[10] Yu L and Skov A L 2018 Macromol. Rapid Commun. 39 1800383

[11] Ito K 2012 Polym. J. 44 38-41

[12] Minato K, Mayumi K, Maeda R, Kato K, Yokoyama $\mathrm{H}$ and Ito K 2017 Polymer (Guildf). 128 386-91

[13] Zhuo Y, Li T, Wang F, Håkonsen V, Xiao S, He J and Zhang Z 2019 Soft Matter 15 3607-11

[14] Du R, Xu Z, Zhu C, Jiang Y, Yan H and Wu H 2020 Adv. Funct. Mater. 301907139

[15] Mayumi K, Liu C, Nakai T, Ishida M, Takeuchi H, Inoue K, Urayama K, Yokoyama H and Ito K 2019 Proc.SPIE 10966

[16] Shintake J, Matsuno K, Baba K and Takeuchi H 2019 Proc.SPIE 10966

[17] Zhou S, Wang J, Wang G, Jiang Z and Ren H 2017 Eur. Polym. J. 90 312-22

[18] Araki J, Kataoka T and Ito K 2008 Soft Matter 4 245-9

[19] Marciniec B 2009 Hydrosilylation: A Comprehensive Review on Recent Advances ed B Marciniec (Dordrecht: Springer Netherlands) pp 159-89

[20] Yu L, Madsen F B and Skov A L 2018 Int. J. Smart Nano Mater. $9217-232$

[21] Araki J 2010 J. Polym. Sci. Part A Polym. Chem. 48 5258-64

[22] Ohmori K, Abu Bin I, Seki T, Liu C, Mayumi K, Ito K and Takeoka Y 2016 Chem. Commun. 52 13757-9

[23] Harada A and Kamachi M 1990 Macromolecules 23 2821-3

[24] Harada A, Li J and Kamachi M 1992 Nature 356 325-7

[25] Ceccato M, Lo Nostro P and Baglioni P 1997 Langmuir 13 2436-8

[26] Inomata A, Sakai Y, Zhao C, Ruslim C, Shinohara Y, Yokoyama H, Amemiya Y and Ito K 2010 Macromolecules 43 4660-6

[27] Araki J and Ito K 2006 J. Polym. Sci. Part A Polym. Chem. 44 6312-23

[28] Shinohara Y, Kayashima K, Okumura Y, Zhao C, Ito K and Amemiya Y 2006 Macromolecules 39 7386-91

[29] Kato K, Hori A and Ito K 2018 Polymer (Guildf). 147 67-73

[30] Inutsuka M, Inoue K, Hayashi Y, Inomata A, Sakai Y, Yokoyama H and Ito K 2015 Polymer (Guildf). 59 10-5

[31] Kato K and Ito K 2011 Soft Matter 7 8737-40

[32] Sommer-Larsen P and Larsen AL 2004 Proc. SPIE 538568 